\title{
A predator-infected prey model with harvesting of infected prey
}

\author{
S.A. Wuhaib*, Y. Abu Hasan \\ School of Mathematical Sciences, Universiti Sains Malaysia, 11800 Penang Malaysia \\ *Corresponding author, e-mail: s_s18600@yahoo.com
}

Received 7 Jan 2013

Accepted 5 Apr 2013

\begin{abstract}
In this paper, we introduce a predator-prey with susceptible and infected prey model. The model includes the harvesting of infected prey. We assume that the predator avoids the infected prey. The susceptible prey becomes infected when they are in contact with infected prey and recover to be susceptible again. We find the equilibrium points and the conditions for their existence and stability. We also show the non-existence of periodic solutions. Numerical simulations explain the effect of the parameters on the behaviour of the three classes of populations. The simulations also give the region of the solution and guarantees that all solutions of the system lie within the region.
\end{abstract}

KEYWORDS: predator-prey, SIS, stability, invariant

\section{INTRODUCTION}

Mathematics is one way to explain many of the ideas and concepts in the sciences. In the field of ecology, a lot of theoretical studies were carried out since the beginning of last century to explain the interaction between the ecological communities. One particular study describes the interaction between one population (prey) and the other (predator) living in a closed environment with the two populations striving for survival. The basic model is known as the LotkaVolterra model. This model was intensively studied and developed to describe more complicated interactions. A survey of important contributions can be found in Hethcote ${ }^{1}$. May and Leonard ${ }^{2}$ constructed $^{2}$ a model to study the effect of infectious diseases in predator-prey systems. In their model, the basic epidemic model was combined with the Lotka-Volterra model. Subsequently, many researchers have studied the effects of a disease in the prey or the predator on the dynamics of the predator prey system ${ }^{3-6}$. In another area of study, researchers have studied the effect of harvesting the prey or the predator on the coexistence of both classes of population ${ }^{7-9}$.

In this paper, we develop a model in which the prey follows the susceptible-infected-susceptible cycle. Within this cycle, the infected prey is harvested and the predators consume the susceptible prey only. There are many documented cases on this, an example is the relationship between aquatic snails and fishes as reported by Holmes et $\mathrm{al}^{10}$. In the following section we describe the model, to be followed by a study on the stability of the equilibrium points. Next, we discuss the nature of the solutions and finally the numerical simulations to support the model.

\section{MATHEMATICAL MODEL}

In this section, we consider the following hypotheses.

(i) The susceptible prey population grows according to the logistic equation with growth rate $r_{1}>0$, and carrying capacity $K>0$. The infected prey grows according the logistic equation with growth rate $r_{2}>0$, and with carrying capacity $L>0$.

(ii) The prey follows the susceptible-infectedsusceptible cycle.

(iii) The harvesting is only for the infected prey.

(iv) There is no other source of food for the predator other than the susceptible prey, if there is no susceptible prey the predator can die.

(v) The predator cannot be infected.

The model can be written as

$$
\begin{aligned}
\frac{\mathrm{d} S}{\mathrm{~d} t} & =r_{1} S\left(1-\frac{S}{K}\right)-\rho S I+\beta I-\gamma S F, \\
\frac{\mathrm{d} I}{\mathrm{~d} t} & =r_{2} I\left(1-\frac{I}{L}\right)+\rho S I-\beta I-q I, \\
\frac{\mathrm{d} F}{\mathrm{~d} t} & =\gamma_{1} S F-d F,
\end{aligned}
$$

with condition $r_{2}>\beta+q$. In system (1), $r_{1}$ and $r_{2}$ are intrinsic growth rate coefficients of susceptible prey species and infected prey, respectively, $K$ and $L$ are their respective carrying capacities, $\gamma$ is the depletion 
rate coefficient of the prey species due to the predator, $\rho$ is the rate of the contact between susceptible prey and infected prey, $\beta$ is the rate of transformation from infected prey to susceptible prey, $q$ is the rate of harvesting of infected prey, $\gamma_{1}$ is the growth rate coefficient of predator due to its interaction with the susceptible prey, where we assume $\gamma>\gamma_{1}$ and $d$ is the natural death rate coefficient of the predator species, also all parameters are positive. System (1) reduces to the standard prey-predator model without $I$ :

$$
\begin{aligned}
\frac{\mathrm{d} S}{\mathrm{~d} t} & =r_{1} S\left(1-\frac{S}{K}\right)-\gamma S F, \\
\frac{\mathrm{d} F}{\mathrm{~d} t} & =\gamma_{1} S F-d F .
\end{aligned}
$$

In this case there are two equilibria, $E(0,0)$ and $\bar{E}(\bar{S}, \bar{F})$, where $\bar{S}=d / \gamma_{1}$, and

$$
\bar{F}=\frac{r}{\gamma_{1}}\left(1-\frac{\bar{S}}{K}\right) .
$$

\section{EQUILIBRIUM AND STABILITY ANALYSIS}

In system (1), there are three equilibrium points. The first two are: $E_{0}(0,0,0)$ and $\tilde{E}(\tilde{S}, \tilde{I}, 0)$, where

$$
\begin{aligned}
& \tilde{S}=\frac{1}{\rho}\left(\beta+q-r_{2}\left(1-\frac{\tilde{I}}{L}\right)\right), \\
& \tilde{I}=\frac{r_{1} \tilde{S}}{\rho \tilde{S}-\beta}\left(1-\frac{\tilde{S}}{K}\right) .
\end{aligned}
$$

The third is $E^{*}=\left(S^{*}, I^{*}, F^{*}\right)$, where $S^{*}=d / \gamma_{1}$,

$$
\begin{aligned}
I^{*} & =\frac{L}{r_{2}}\left(r_{2}+\rho S^{*}-(\beta+q)\right), \\
F^{*} & =\frac{1}{\gamma}\left(r_{1}-\frac{r_{1} S^{*}}{K}-\rho I^{*}+\frac{\beta I^{*}}{S^{*}}\right),
\end{aligned}
$$

with condition $\left(\gamma_{1} \beta>\rho d\right)$. The Jacobian matrix of system (1) has entries

$$
\begin{aligned}
& A_{11}=r_{1}\left(1-\frac{2 S}{K}\right)-\rho I+\gamma F \\
& A_{12}=-\rho S+\beta \\
& A_{13}=-\gamma S \\
& A_{21}=\rho I \\
& A_{22}=r_{2}\left(1-\frac{2 I}{L}\right)+\rho S-\beta-q, \\
& A_{23}=A_{32}=0 \\
& A_{31}=\gamma_{1} F \\
& A_{33}=\gamma_{1} S-d .
\end{aligned}
$$

The eigenvalues of equilibrium point $E_{0}$ are $r_{1}>0$, $r_{2}-(\beta+q)>0$, and $-d<0$. Thus they are always unstable. The eigenvalues of $\tilde{E}$ are $\gamma_{1} \tilde{S}-d$ and

$$
\frac{1}{2}\left[r_{1}\left(1-\frac{2 \tilde{S}}{K}\right)-\tilde{I}\left(\rho+\frac{r_{2}}{L}\right) \pm D^{\frac{1}{2}}\right],
$$

where

$$
\begin{aligned}
D= & {\left[r_{1}\left(1-2 \frac{\tilde{S}}{K}\right)-\tilde{I}\left(\rho+\frac{r_{2}}{L}\right)\right]^{2} } \\
& +4 \rho \tilde{I}\left[\frac{r_{2}}{\rho L}\left(r_{1}\left(1-\frac{2 \tilde{S}}{K}\right)-\rho \tilde{I}\right)-\rho \tilde{S}+\beta\right] .
\end{aligned}
$$

The characteristic equation in the case of $E^{*}$ is

$$
\lambda^{3}+A \lambda^{2}+B \lambda+C=0,
$$

where

$$
\begin{gathered}
A=\left(r_{2}+\rho S^{*}-(\beta+q)\right)+\frac{r_{1} S^{*}}{K}+\frac{\beta I^{*}}{S^{*}}>0, \\
B=\left(\frac{r_{1} S^{*}}{K}+\frac{\beta I^{*}}{S^{*}}\right)\left(r_{2}+\rho S^{*}-(\beta+q)\right) \\
\quad+\gamma \gamma_{1} S^{*} F^{*}+\left(\rho S^{*}-\beta\right) \rho I^{*}, \\
C=\left(r_{2}+\rho S^{*}-(\beta+q)\right)\left(\gamma \gamma_{1} S^{*} F^{*}\right)>0,
\end{gathered}
$$

and $A B>C$. From the Routh-Hurwitz criteria $E^{*}$ is locally stable.

Lemma 1 The equilibrium point $E^{*}$ is globally stable.

Proof: Let

$$
\begin{array}{r}
V=\left(S-S^{*}-S^{*} \ln \frac{S}{S^{*}}\right)+c_{1}\left(I-I^{*}-I^{*} \ln \frac{I}{I^{*}}\right) \\
+c_{2}\left(F-F^{*}-F^{*} \ln \frac{F}{F^{*}}\right) .
\end{array}
$$

If $c_{2}=\gamma / \gamma_{1}$ and $c_{1}=1$, we get

$$
\begin{aligned}
\frac{\mathrm{d} V}{\mathrm{~d} t}=\frac{r_{1}}{K}\left(S-S^{*}\right)^{2} & -\frac{r_{2}}{L}\left(I-I^{*}\right)^{2} \\
& -\beta\left(\frac{I^{*}}{S^{*}}-\frac{I}{S}\right)\left(S-S^{*}\right)<0,
\end{aligned}
$$

if $I^{*} S>S^{*} I$, then $E^{*}$ is globally stable under this condition.

\section{INVARIANT REGION}

In this section, we consider conditions for the coexistence of the three classes of populations. There are four cases due to the maximum size of population of both classes $K$ and $L$ : 
(i) $S=K, I=L, F>0$,

(ii) $S=K, I<L, F>0$,

(iii) $S<K, I=L, F>0$,

(iv) $S<K, I<L, F>0$.

In the first case, system (1) becomes as

$$
\begin{aligned}
\frac{\mathrm{d} S}{\mathrm{~d} t} & =-\rho K L+\beta L-\gamma K F, \\
\frac{\mathrm{d} I}{\mathrm{~d} t} & =\rho K L-\beta L-q L, \\
\frac{\mathrm{d} F}{\mathrm{~d} t} & =\gamma_{1} K F-d F .
\end{aligned}
$$

The interior region $R_{+}^{3}=\left(K, L, F^{*}\right)$, where

$$
F^{*}=\frac{L}{\gamma K}(\beta-\rho K),
$$

with condition $\beta>\rho K$. In the second case, when $S=K, I<L$. The system (1) becomes as

$$
\begin{aligned}
\frac{\mathrm{d} S}{\mathrm{~d} t} & =-\rho K I+\beta I-\gamma K F, \\
\frac{\mathrm{d} I}{\mathrm{~d} t} & =r_{2} I\left(1-\frac{I}{L}\right)+\rho K I-\beta I-q I, \\
\frac{\mathrm{d} F}{\mathrm{~d} t} & =\gamma_{1} K F-d F .
\end{aligned}
$$

The interior region $R_{+}^{3}=\left(K, I^{*}, F^{*}\right)$, where

$$
r_{2}\left(1-\frac{I}{L}\right)+\rho K-\beta-q=0 .
$$

Thus

$$
\begin{aligned}
I^{*} & =\frac{L}{r_{2}}\left(r_{2}+\rho K-(\beta+q)\right), \\
F^{*} & =\frac{L}{r_{2} \gamma K}(\beta-\rho K)\left(r_{2}+\rho K-(\beta+q)\right),
\end{aligned}
$$

with necessary condition $\beta>\rho K$. In the third case, when $S<K, I=L$ The system (1) becomes as

$$
\begin{aligned}
\frac{\mathrm{d} S}{\mathrm{~d} t} & =r_{1} S\left(1-\frac{S}{K}\right)-\rho S L+\beta L-\gamma S F, \\
\frac{\mathrm{d} I}{\mathrm{~d} t} & =\rho S L-\beta L-q L, \\
\frac{\mathrm{d} F}{\mathrm{~d} t} & =\gamma_{1} S F-d F .
\end{aligned}
$$

The interior region $R_{+}^{3}=\left(S^{*}, L, F^{*}\right)$, where

$$
\begin{aligned}
S^{*} & =\frac{\beta+q}{\rho}=\frac{d}{\gamma_{1}}, \\
F^{*} & =\frac{1}{\gamma}\left(r_{1}\left(1-\frac{S^{*}}{K}\right)-\frac{q L}{S^{*}}\right) .
\end{aligned}
$$

In the fourth case, when $S<K, I<L$. The system (1) becomes as

$$
\begin{aligned}
\frac{\mathrm{d} S}{\mathrm{~d} t} & =r_{1} S\left(1-\frac{S}{K}\right)-\rho S I+\beta I-\gamma S F, \\
\frac{\mathrm{d} I}{\mathrm{~d} t} & =r_{2} I\left(1-\frac{I}{L}\right)+\rho S I-\beta I-q I, \\
\frac{\mathrm{d} F}{\mathrm{~d} t} & =\gamma_{1} S F-d F .
\end{aligned}
$$

The interior region $R_{+}^{3}=\left(S^{*}, I^{*}, F^{*}\right)$, where

$$
\begin{aligned}
S^{*} & =\frac{d}{\gamma_{1}}, \\
I^{*} & =\frac{L}{r_{2}}\left(r_{2}+\rho S-(\beta+q)\right), \\
F^{*} & =\frac{1}{\gamma S^{*}}\left(r_{1} S^{*}\left(1-\frac{S^{*}}{K}\right)-\rho S^{*} I^{*}+\beta I^{*}\right),
\end{aligned}
$$

with condition $\beta \gamma_{1}>\rho d$. The equilibrium $E^{*}$ of the system (1) is the interior. Thus this equilibrium must exist in the intersection of these areas. Therefore the invariant region of system (1) is the intersection of all these areas.

\section{PROPERTIES OF SOLUTION}

In this section, we discuss the solution of the system (1) when it is bounded, positive, and not periodic.

Lemma 2 The solution of system (1) is bounded and positive.

Proof: First we define the function $w(t)=S(t)+$ $I(t)+F(t)$. Then

$$
\frac{\mathrm{d} w}{\mathrm{~d} t}+\mu w(t)=\frac{\mathrm{d} S}{\mathrm{~d} t}+\frac{\mathrm{d} I}{\mathrm{~d} t}+\frac{\mathrm{d} F}{\mathrm{~d} t}+\mu S+\mu I+\mu F .
$$

We assume $0<\mu<d$. Since $\gamma>\gamma_{1}$, we get

$\frac{\mathrm{d} w}{\mathrm{~d} t}+\mu w(t) \leqslant\left(\frac{K\left(r_{1}+\mu\right)}{2 r_{1}}\right)^{2}+\left(\frac{L\left(r_{2}+\mu\right)}{2 r_{2}}\right)^{2}=v$.

Then

$\frac{\mathrm{d} w}{\mathrm{~d} t}+\mu w(t) \leqslant v$

$0<w(S, I, F) \leqslant \frac{v}{\mu}\left(1-\mathrm{e}^{-\mu t}\right)+\left.\mathrm{e}^{-\mu t}(S, I, F)\right|_{t=0}$.

This means that the solutions are bounded and positive.

Lemma 3 The system (1) has no periodic solution. 


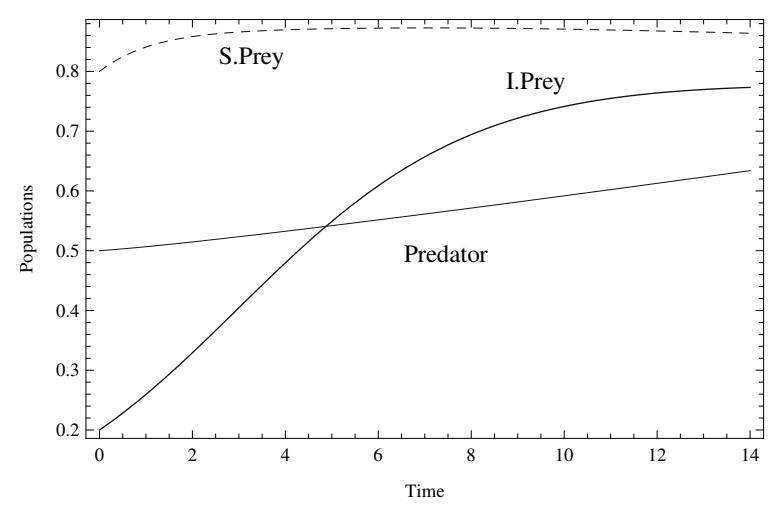

Fig. 1 The low harvesting leads to an increase in the infected prey.

Proof: For no periodic orbit in this system, we use Dulac's criterion and consider the $S-I$ plane. Let

$$
\begin{aligned}
& H(S, I)=\frac{1}{S I} \\
& h_{1}(S, I)=r_{1} S\left(1-\frac{S}{K}\right)-\rho S I+\beta I-\gamma S F, \\
& h_{2}(S, I)=r_{2} I\left(1-\frac{I}{L}\right)+\rho S I-\beta I-q I .
\end{aligned}
$$

Then

$$
\Delta(S I)=\frac{\partial\left(h_{1} H\right)}{\partial S}+\frac{\partial\left(h_{2} H\right)}{\partial I} .
$$

Hence

$$
\Delta(S I)=-\frac{r_{1}}{K I}-\frac{\beta}{S^{2}}-\frac{r_{2}}{S L} .
$$

It is clear that there is no change in sign, therefore this system cannot have any periodic solution in $S-I$ plane. Also we can show in the $S-F$ plane that there is no change in sign, so no periodic in $S-F$ plane. Hence the system has no periodic solution.

\section{NUMERICAL SIMULATION}

In this section, we discuss the effect of effort of harvest on the disease. First, we fixed all parameters to ensure all populations survive. Then we find the effect of harvest on the disease.

When $\rho=\beta$, we take these two parameters to be large. We noticed that the infected prey increases when there is a low harvest (Fig. 1), and decreases when there is a large harvest (Fig. 2). When $\rho=$ $\beta$, the two parameters are small (Fig. 3 and Fig. 4). When $\rho>\beta$, the infected prey increases (Fig. 5 and Fig. 6) but this increase also depends on the amount of harvest. Unlike the second case, i.e., $\beta>\rho$, in this case the infected prey decreases if the harvest is large (Fig. 7) or the harvest is low (Fig. 8).

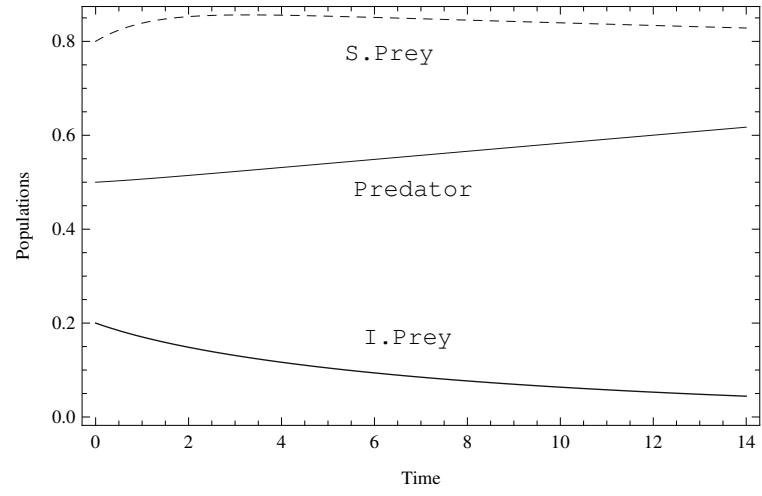

Fig. 2 The high harvesting leads to a decrease in the infected prey.

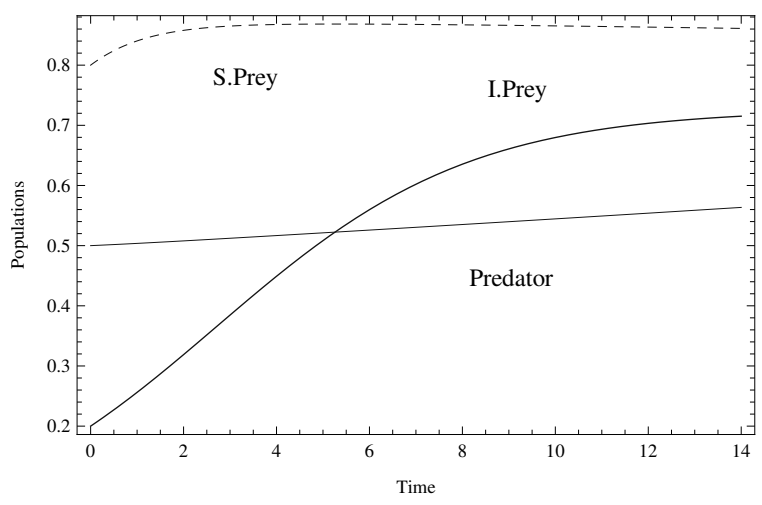

Fig. 3 The low harvesting leads to an increase in the infected prey.

\section{CONCLUSIONS}

A predator-prey model, where the prey followed the susceptible-infected-susceptible cycle, was developed. In order to maintain a healthy population, the

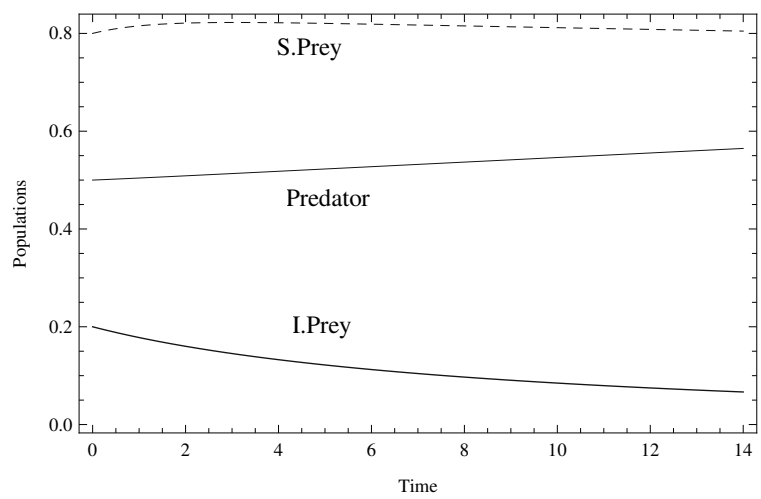

Fig. 4 The high harvesting leads to a decrease in the infected prey. 


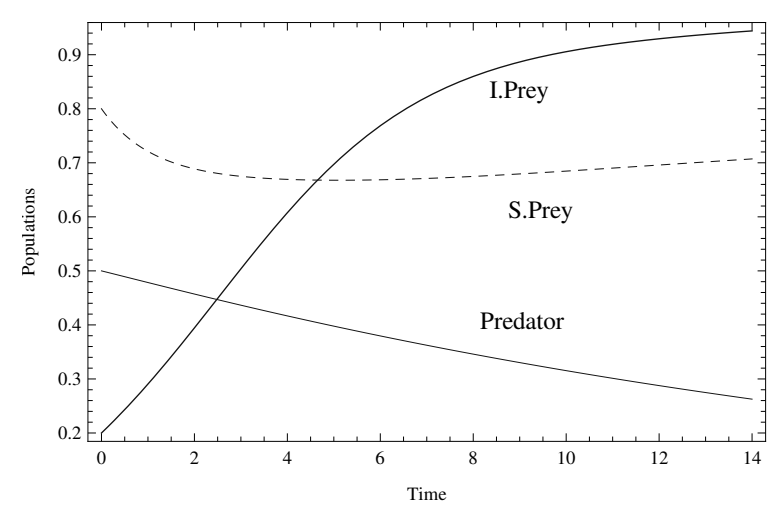

Fig. 5 The infected prey increasing for $\rho>\beta, q=0.1$.

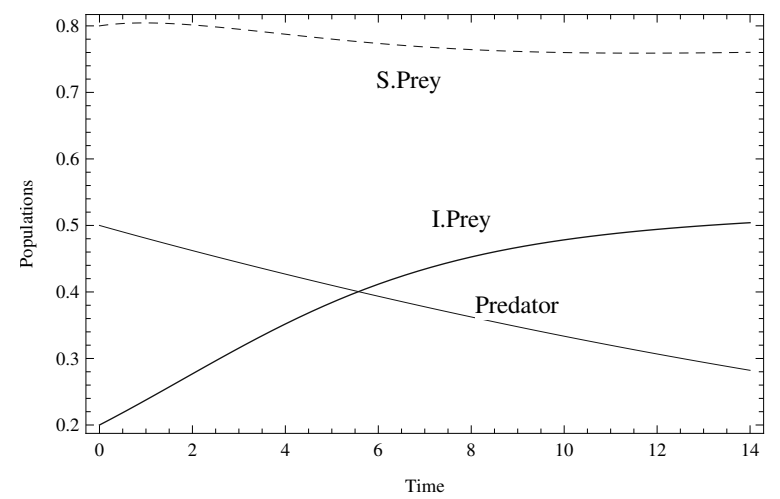

Fig. 6 The infected prey increasing for $\rho>\beta, q=0.5$.

infected prey was harvested. To maintain a balance between the prey and the predator, the harvesting rate has to be fine-tuned as a result of the rate of infection and recovery. Conditions for stability of the equilibrium points for the two populations were obtained. Obtained also were the region of the solutions, where the solutions are bounded. It is also observed that the increase in harvest affects the disease and thus

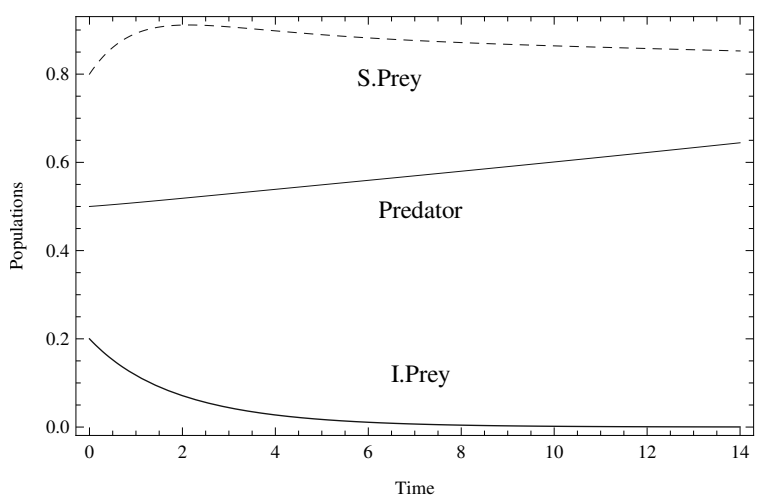

Fig. 7 The infected prey decreasing for $\beta>\rho, q=0.5$.

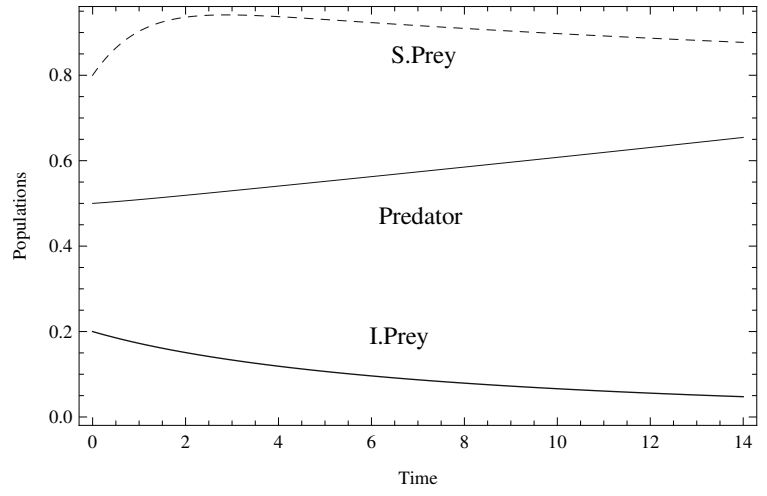

Fig. 8 The infected prey decreasing for $\beta>\rho, q=0.1$.

prevents the occurrence of an epidemic.

Acknowledgements: This study was fully supported by a grant from the School of Mathematical sciences, Universiti Sains Malaysia, 11800 Penang Malaysia.

\section{REFERENCES}

1. Hethcote $\mathrm{H}$ (2000) The mathematics of infectious diseases. SIAM Rev 42, 599-653.

2. May R, Leonard W (1975) Nonlinear aspects of competition between three species. SIAM J Appl Math 29, 243-53.

3. Venturino E (2002) Epidemics in predator-prey models: disease in the predators. Math Med Biol 19, 185-205.

4. Hethcote H, Wang W, Han L, Ma Z (2004) A predatorprey model with infected prey. Theor Popul Biol 66, 259-68.

5. Haque M, Venturino E (2006) The role of transmissible diseases in the Holling-Tanner predator-prey model. Theor Popul Biol 70, 273-88.

6. Haque M, Venturino E (2007) An ecoepidemiological model with disease in predator: the ratio-dependent case. Math Meth Appl Sci 30, 1791-809.

7. Dubey B, Chandra P, Sinha P (2003) A model for fishery resource with reserve area. Nonlinear Anal $R$ World Appl 4, 625-37.

8. Zhang X, Chen L, Neumann A (2000) The stagestructured predator-prey model and optimal harvesting policy. Math Biosci 168, 201-10.

9. Chakraborty S, Pal S, Bairagi N (2011) Predator-prey interaction with harvesting: mathematical study with biological ramifications. Appl Math Model 36, 4055-9.

10. Holmes J, Bethel W (1972) Modification of intermediate host behaviour by parasites. In: Canning EU, Wright CA (eds) Behavioural Aspects of Parasite Transmission, Academic Press, New York, pp 123-49. 